\title{
RESCALINGS AT POSSIBLE SINGULARITIES OF NAVIER-STOKES EQUATIONS IN HALF-SPACE
}

\author{
G. SEREGIN AND V. ŠVERÁK
}

\begin{abstract}
The relationship is clarified between a possible blow-up for strong solutions of the initial boundary value problem for the incompressible Navier-Stokes equations in $\left\{x_{3}>0\right\}$, and the Liouville theorem for mild bounded ancient solutions.
\end{abstract}

\section{$\S 1$. INTRODUCTION}

Consider the initial boundary-value problem for the incompressible Navier-Stokes equations in half-space $\mathbb{R}_{+}^{3}=\left\{x_{3}>0\right\}$ :

$$
\left.\begin{array}{rlrl}
\partial_{t} v+v \cdot \nabla v-\Delta v & =-\nabla q \\
\operatorname{div} v & =0
\end{array}\right\} \quad \begin{array}{ll}
\text { in } \mathbb{R}_{+}^{3} \times(0, \infty), \\
\left.v(\cdot, t)\right|_{\partial \mathbb{R}_{+}^{3}}=0 & \text { for } t>0, \\
v(\cdot, 0)=v_{0} & \text { in } \mathbb{R}^{3},
\end{array}
$$

where $v_{0}$ is a sufficiently regular div-free field in $\mathbb{R}_{+}^{3}$ with $\left.v_{0}\right|_{\partial \mathbb{R}_{+}^{3}}=0$ and with sufficiently fast decay as $x \rightarrow \infty$.

Our main goal is to understand the relationship between a possible blow-up for strong solutions and the Liouville theorem for mild bounded ancient solutions, in the spirit considered in [6] for the entire space. We recall that the local-in-time existence of strong solutions was proved in the entire space in [9], and for bounded domains similar results appeared in [5] As to unbounded domains, we refer, for example, to [8] or [2].

Consider the local-in-time strong solution $v$ on its maximal interval of existence $[0, T)$, which will be assumed finite. The time $T$ is then the blow-up time. It is known that such solutions are unique (unlike the weak solutions, which on the other hand are global). We set

$$
g(t)=\sup _{0<\tau \leq t} M(\tau)
$$

where

$$
M(t)=\sup _{x \in \mathbb{R}_{+}^{3}}|v(x, t)|
$$

It is well known that $g(t) \rightarrow+\infty$ as $t \rightarrow T_{-}$, see for example 9 .

Our considerations are motivated by methods used in the theory of geometric flows and based on scale-invariant transformations of the solution $v$ when time is approaching $T$, see [6] for a more detailed discussion and references. In the case of the NavierStokes equations, the scale-invariant transformations have the form $v(x, t), q(x, t) \rightarrow$ $\lambda v\left(\lambda x, \lambda^{2} t\right), \lambda^{2} q\left(\lambda x, \lambda^{2} t\right)$. In the entire space, taking limits of sequences of suitably

2010 Mathematics Subject Classification. Primary 35Q30, 76D05.

Key words and phrases. Incompressible Navier-Stokes equations, blow-up, mild bounded ancient solution.

${ }^{1}$ At the time of this writing it is unknown whether such solutions exist globally. 
scaled solutions produces so-called bounded ancient (backward) solutions of the NavierStokes equations. They are defined on the semiinfinite time interval $]-\infty, 0]$ (backward in time), are bounded in $L_{\infty}$, and in fact belong to a subclass called mild bounded ancient solutions. In [6], it was shown that mild bounded ancient solutions are infinitely smooth both in space and in time. Moreover, if $T$ is a blow-up time, the limiting mild bounded ancient solution cannot vanish. It was conjectured in 6 , that any mild bounded ancient solution is a constant. This would rule out blow-up of Type I in the case of the Cauchy problem (whole space). We recall that Type I blow-up is usually defined by the inequality

$$
|v(x, t)| \leq \frac{c}{\sqrt{T-t}}
$$

for any $x \in \mathbb{R}^{3}$ and any $t<T$, although more general definitions are also possible.

To show a difference between bounded ancient solutions in the whole space and in the half-space, we recall their definitions. We say that $u$ is a bounded ancient solution of the Navier-Stokes equations if $u$ is bounded in $\left.Q_{-}=\mathbb{R}^{3} \times\right]-\infty, 0[$ and satisfies these equations in the sense of distributions with divergence free test functions, i.e.,

$$
\int_{Q_{-}}\left(u \cdot\left(\partial_{t} \varphi+\Delta \varphi\right)+u \otimes u: \nabla \varphi\right) d z=0
$$

for any $\varphi \in C_{0,0}^{\infty}\left(Q_{-}\right):=\left\{\varphi \in C_{0}^{\infty}\left(Q_{-}\right): \operatorname{div} \varphi=0\right\}$;

$$
\int_{Q_{-}} u \cdot \nabla q d z=0
$$

for any $q \in C_{0}^{\infty}\left(Q_{-}\right)$. By scaling, we may assume that $|u|$ is bounded by one. There are simple nontrivial bounded ancient solutions of the form

$$
u(x, t)=a(t)
$$

where $a$ is an arbitrary bounded function of $t$ only.

A vector field $u$ is called a mild bounded ancient solution if $u$ is a bounded ancient solution and there exists a pressure field $p \in L_{\infty}\left(-\infty, 0 ; B M O\left(\mathbb{R}^{3}\right)\right)$ such that

$$
\int_{Q_{-}}\left(u \cdot\left(\partial_{t} \varphi+\Delta \varphi\right)+u \otimes u: \nabla \varphi\right) d z=-\int_{Q_{-}} p \operatorname{div} \varphi d z
$$

for any $\varphi \in C_{0}^{\infty}\left(Q_{-}\right)$. It is not so difficult to see that any solution of the form (1.8) is a mild bounded ancient solution if and only if $a(t)=$ constant. As has already been mentioned, mild bounded ancient solutions are infinitely smooth and have the following property: for any $A<0$, they can be presented in the form

$$
\begin{aligned}
u_{i}(x, t) & =\int_{\mathbb{R}^{3}} \Gamma(x-y, t-A) u_{i}(y, A) d y \\
& +\int_{A}^{t} \int_{\mathbb{R}^{3}} K_{i j m}(x, y, t-\tau) u_{j}(y, \tau) u_{m}(y, \tau) d y d \tau,
\end{aligned}
$$

where $\Gamma$ is the well known heat kernel and $K$ is obtained from the Oseen tensor by differentiation in spatial variables, see details in [14] and [6]. That is why those solutions are said to be mild. By the way, this is the original definition of mild bounded ancient solutions given in [6], and the equivalent definition in terms of the pressure appeared in [12] later on.

The case of the half-space is more complicated. First, it is not immediately clear how to understand the homogeneous Dirichlet boundary conditions when the velocity $u$ is 
only bounded in $Q_{-}^{+}:=\left\{z=(x, t): x \in \mathbb{R}_{+}^{3},-\infty<t<0\right\}$. We shall use the following weak definition of (1.2):

$$
\int_{Q_{-}^{+}}\left(u \cdot\left(\partial_{t} \varphi+\Delta \varphi\right)+u \otimes u: \nabla \varphi\right) d z=0
$$

for any $\varphi \in C_{0,0}^{\infty}\left(Q_{-}\right)$with $\varphi\left(x^{\prime}, 0, t\right)=0$ for any $x^{\prime} \in \mathbb{R}^{2}$ and for any $-\infty<t<0$;

$$
\int_{Q_{-}^{+}} u \cdot \nabla q d z=0
$$

for any $q \in C_{0}^{\infty}\left(Q_{-}\right)$.

In [3] and [4, a class of simple nontrivial bounded ancient solutions to the NavierStokes equations was presented. They describe a bounded shear flow in the half-space and have the form

$$
u(x, t)=\left(u_{1}\left(x_{3}, t\right), u_{2}\left(x_{3}, t\right), 0\right) .
$$

It was also shown in [3] and [4] that there are no other nontrivial solutions to the linear Stokes system in the half-space. It is unknown whether or not this is true for the nonlinear case.

We now define mild bounded ancient solutions in a half-space.

Definition 1.1. A bounded function $u$ is a mild bounded ancient solution if and only if there exists a pressure $p$ such that $p=p^{1}+p^{2}$, where the even extension of $p^{1}$ to the whole $\mathbb{R}^{3}$ with respect to $x_{3}$ is an $L_{\infty}\left(-\infty, 0 ; B M O\left(\mathbb{R}^{3}\right)\right)$-function,

$$
\Delta p^{1}=-\operatorname{div} \operatorname{div} u \otimes u
$$

in $Q_{-}^{+}$with $p_{, 3}^{1}\left(x^{\prime}, 0, t\right)=0$, and $p^{2}(\cdot, t)$ is a harmonic function in $\mathbb{R}_{+}^{3}$ whose gradient satisfies the estimate

$$
\left|\nabla p^{2}(x, t)\right| \leq c \ln \left(2+1 / x_{3}\right)
$$

for all $(x, t) \in Q_{-}^{+}$and has the property

$$
\sup _{x^{\prime} \in \mathbb{R}^{2}}\left|\nabla p^{2}(x, t)\right| \rightarrow 0
$$

as $x_{3} \rightarrow \infty ; u$ and $p$ satisfy (1.12) and

$$
\int_{Q_{-}^{+}}\left(u \cdot\left(\partial_{t} \varphi+\Delta \varphi\right)+u \otimes u: \nabla \varphi+p \operatorname{div} \varphi\right) d x d t=0
$$

for any $\varphi \in C_{0}^{\infty}\left(Q_{-}\right)$with $\varphi\left(x^{\prime}, 0, t\right)=0$ for any $x^{\prime} \in \mathbb{R}^{2}$ and for any $t<0$.

Remark 1.2. If $u$ is a mild bounded ancient solution, then $\nabla u \in L_{\infty}\left(Q_{-}^{+}\right)$. The function $u$ is infinitely smooth in spatial variables in the upper half-space $x_{3}>0$.

As in the case of the entire space, mild bounded ancient solutions can be defined by formula (1.10), in which $\mathbb{R}^{3}$ is replaced with $\mathbb{R}_{+}^{3}$ and kernels with their half-space analogs, see $\S 2$ for details. The corresponding statement might be called the equivalence theorem. The proof of such a result is more involved than its whole space version and will be published elsewhere.

It should be noticed that a nontrivial solution of the form (1.13) is a mild bounded ancient solutions if and only $u=0$. Indeed, for solutions (1.13), the gradient of the pressure is a function of time only. By the above theorem, this is only possible if the gradient of the pressure is equal to zero. In its turn, this means that each component $u_{\alpha}, \alpha=1,2$, is a bounded ancient solution to the heat equation in $\left.\left\{x_{3}>0\right\} \times\right]-\infty, 0[$ with the boundary condition $u_{\alpha}(0, t)=0$, which implies that $u_{\alpha} \equiv 0$.

We believe that the following is true. 
Conjecture 1.3. There is no nontrivial mild bounded ancient solution of the NavierStokes equations in the half-space.

The validity of Conjecture 1.3 and the conjecture, made in [6] and mentioned above, would rule out Type I blow-up in the broad sense, when understood as blow-up solutions with a suitable bounded scale-invariant quantity.

We state our main result.

Theorem 1.4. Assume that initial boundary value problem (1.1)-(1.3) has a solution that admits blow-up at time $T$. There exists at least one nontrivial (nonzero) mild bounded ancient solution either in the whole space or in the half-space.

The appearance of mild bounded ancient solutions in the whole space is not surprising and should be expected if the solution $v$ to the original problem (1.1)-(1.3) is smooth near the boundary $x_{3}=0$ near the blow-up time $T$. This scenario of blow-up can be called an interior blowup. All other mild bounded ancient solutions are then related to a boundary blowup. We note that a boundary blow-up could still lead to a mild bounded ancient solution in the whole space. This might happen when the velocity tends to infinity fast in comparison with the rate at which $x$ is approaching the boundary.

An interesting consequence of the above theorem is the following statement.

Proposition 1.5. There exists $\varepsilon>0$ (independent of $v$ ) such that if

$$
|v(x, t)| \leq \frac{\varepsilon}{x_{3}}
$$

for all $x \in \mathbb{R}_{+}^{3}$ and $\left.t \in\right] 0, T[$, the solution $v$ does not blow-up.

\section{§2. Prelimaries}

Given $A<0$, consider the following Stokes problem in half-space:

$$
\partial_{t} u-\Delta u+\nabla p=-\operatorname{div} F, \quad \operatorname{div} u=0
$$

in $\left.\mathbb{R}_{+}^{3} \times\right] A, 0[$,

for $\left.\left(x^{\prime}, t\right) \in \mathbb{R}^{2} \times\right] A, 0[$,

$$
u\left(x^{\prime}, 0, t\right)=0
$$

for $x \in \mathbb{R}_{+}^{3}$.

$$
u(x, A)=u_{0}(x)
$$

In addition, we assume that $u_{0}$ is divergence free and $F$ and its first derivatives vanish at the boundary $x_{3}=0$. If $F$ and $u_{0}$ are sufficiently smooth and decay sufficiently fast at infinity, a solution of the above problem can be presented in the following way, see [13,

$$
u=u^{1}+u^{2}
$$

where

$$
u^{1}(x, t)=\int_{\mathbb{R}_{+}^{3}} G(x, y, t-A) u_{0}(y) d y
$$

for any $x \in \mathbb{R}_{+}^{3}$ and any $\left.t \in\right] A, 0[$, and

$$
u^{2}(x, t)=-\int_{A}^{t} \int_{\mathbb{R}_{+}^{3}} G(x, y, t-\tau) \operatorname{div} H(y, \tau) d y d \tau
$$

for the same $x$ and $t$, where $H=F+p^{1} \mathbb{I}$ and $p^{1}$ is a solution of the following Neumann boundary value problem:

$$
\Delta p^{1}=-\operatorname{div} \operatorname{div} F
$$


in $R_{+}^{3}$ and $p_{, 3}^{1}=0$ at $x_{3}=0$. Here, $G$ is the Green function for the Stokes system in the half-space that was studied in [13] and has the form

$$
G=G^{1}+G^{2}
$$

where

$$
\begin{aligned}
& G_{i j}^{1}(x, y, t)=\delta_{i j}\left(\Gamma(x-y, t)-\Gamma\left(x-y^{*}, t\right)\right), \\
& G_{i \beta}^{2}(x, y, t)=4 \frac{\partial}{\partial x_{\beta}} \int_{0}^{x_{3}} \int_{\mathbb{R}^{2}} \frac{\partial E}{\partial x_{i}}(x-z) \Gamma\left(z-y^{*}, t\right) d z, \quad G_{i 3}^{2}(x, y, t)=0 .
\end{aligned}
$$

As in the case of the entire space, it is convenient to present the function $u^{2}$ in the following equivalent way:

$$
u_{i}^{2}(x, t)=\int_{A}^{t} \int_{\mathbb{R}_{+}^{3}} K_{i j m}(x, y, t-\tau) F_{j m}(y, \tau) d y d \tau
$$

for the same $x$ and $t$ as above. The kernel $K$ was introduced in [14] and has the following structure:

$$
K_{i s m}(x, z, t)=\bar{K}_{i s m}(x, z, t)+\widehat{K}_{i s m}(x, z, t),
$$

where $\bar{K}_{i s m}(x, z, t)$ is a linear combination of the terms

$$
\frac{\partial G_{i j}}{\partial z_{k}}(x, z, t)
$$

and $\widehat{K}_{i s m}(x, z, t)$ is a linear combination of the terms

$$
\frac{\partial^{2}}{\partial x_{\alpha} \partial x_{\beta}} \int_{\mathbb{R}_{+}^{3}} G_{i j}(x, y, t) \frac{\partial N^{( \pm)}}{\partial y_{s}}(y, z) d y .
$$

Here, $N^{( \pm)}(x, y)=E(x-y) \pm E\left(x-y^{*}\right)$ with $y^{*}=\left(y^{\prime},-y_{3}\right)$ and $E(x)$ is the fundamental solution of the Laplace equation in $\mathbb{R}^{3}$.

We outline how the above transformations can be done. Our arguments slightly differ from those used in the papers [7] and [14 mentioned above. Consider the following boundary value problems:

$$
\Delta_{y} \Phi_{m n}(x, y, t)=G_{m n}(x, y, t)
$$

with $\partial \Phi_{m n} / \partial y_{3}(x, y, t)=0$ if $n<3$ and with $\Phi_{m n}(x, y, t)=0$ if $n=3$ at $y_{3}=0$. Then integration by parts gives

$$
K_{m j s}(x, y, t)=\frac{\partial^{3} \Phi_{m j}}{\partial y_{i} \partial y_{i} \partial y_{s}}(x, y, t)-\frac{\partial^{3} \Phi_{m n}}{\partial y_{n} \partial y_{j} \partial y_{s}}(x, y, t)
$$

The above splitting for the potential $K$ can easily be derived with help of the initial boundary value problems for function $\Phi$, see (2.7), and some elementary properties of the Green functions $G, N^{+}$, and $N^{-}$.

The following estimates for $G^{i}$ and $\widehat{K}$ were obtained in the papers [13, 14, and [15]:

$$
\begin{aligned}
\left|\frac{\partial^{|\alpha|+|\gamma|} G^{2}}{\partial x^{\alpha} \partial y^{\gamma}}(x, y, t-A)\right| \leq & c(\alpha, \gamma)(t-A)^{-\frac{\gamma_{3}}{2}}\left(t-A+x_{3}^{2}\right)^{-\frac{\alpha_{3}}{2}} \\
& \times\left(\left|x-y^{*}\right|^{2}+t-A\right)^{-\frac{3+\left|\alpha^{\prime}\right|+\left|\gamma^{\prime}\right|}{2}} \exp \left(-\frac{c y_{3}^{2}}{t-A}\right),
\end{aligned}
$$


where $\alpha^{\prime}=\left(\alpha_{1}, \alpha_{2}\right), \gamma^{\prime}=\left(\gamma_{1}, \gamma_{2}\right)$, and $|\gamma|=0,1$,

$$
\begin{aligned}
& \left|\frac{\partial G_{i j}^{1}}{\partial y_{i}}(x, y, t)\right|+\left|\hat{K}_{i s m}(x, y, t)\right| \leq \frac{c}{\left(|x-y|^{2}+t\right)^{2}}, \\
& \left|\partial_{t}^{l} G^{2}(x, y, t)\right| \leq \frac{c}{t^{l}\left(\left|x^{\prime}-y^{\prime}\right|^{2}+x_{3}^{2}+y_{3}^{2}+t\right)^{\frac{3}{2}}} \exp \left(-\frac{c y_{3}^{2}}{t}\right)
\end{aligned}
$$

for $l=0,1$.

Let $K^{1}$ and $K^{2}$ be generated by $G^{1}$ and $G^{2}$, respectively. In particular, we need the following estimate:

$$
\left|\widehat{K}^{2}(x, y, t)\right| \leq \frac{c}{\left(\left|x-y^{*}\right|^{2}+t\right)^{2}},
$$

which can be obtained by an elementary modification of the arguments used in the proof of Proposition 3.1 in [14.

\section{§3. SCALING}

It is not difficult to show that there exists a sequence $\left(x^{(k)}, t_{k}\right)$ with $x^{(k)} \in \mathbb{R}_{+}^{3}$ such that $t_{k} \rightarrow T-0$ and

$$
g\left(t_{k}\right)=M\left(t_{k}\right)=\left|v\left(x^{(k)}, t_{k}\right)\right| \rightarrow \infty .
$$

Remark 3.1. From [1] and [10, it follows that the sequence $x^{(k)}$ is bounded.

We let $M_{k}=M\left(t_{k}\right)$. There are two main scenarios. In the first of the them

$$
x_{3}^{(k)} M_{k} \rightarrow \infty
$$

and we scale $v$ and $q$ so that

$$
u^{(k)}(y, s)=\frac{1}{M_{k}} v(x, t), \quad p_{k}(y, s)=\frac{1}{M_{k}^{2}} q(x, t),
$$

where

$$
y=M_{k}\left(x-x^{(k)}\right), \quad s=M_{k}^{2}\left(t-t_{k}\right) .
$$

By the above scaling, (1.1) and (1.2) are transformed into

$$
\partial_{s} u^{(k)}+u^{(k)} \cdot \nabla u^{(k)}-\Delta u^{(k)}=-\nabla p_{k}, \quad \operatorname{div} u^{(k)}=0
$$

in $\left.Q^{k}:=\left\{y=\left(y^{\prime}, y_{3}\right): y^{\prime} \in \mathbb{R}^{2}, y_{3}>-x_{3}^{(k)} M_{k}\right\} \times\right]-t_{k} M_{k}^{2}, 0[$,

$$
u^{(k)}\left(y^{\prime},-x_{3}^{(k)} M_{k}, t\right)=0
$$

for any $y^{\prime} \in \mathbb{R}^{2}$ and $\left.s \in\right]-t_{k} M_{k}^{2}, 0[$. And, moreover, by (3.1), we have

$$
\left|u^{(k)}(0)\right|=1 \text {. }
$$

In the second scenario,

$$
x_{3}^{(k)} M_{k} \rightarrow a \in[0, \infty[.
$$

This suggests the same scaling (3.3) but with a slightly different change of variables:

$$
y^{\prime}=M_{k}\left(x^{\prime}-x^{(k)}\right), \quad y_{3}=M_{k} x_{3}, \quad s=M_{k}^{2}\left(t-t_{k}\right) .
$$

In this case, (1.1) and (1.2) are transformed into system (3.5), which is valid in

$$
\left.\mathbb{R}_{+}^{3} \times\right]-t_{k} M_{k}^{2}, 0[
$$

and into the boundary condition

$$
u^{(k)}\left(y^{\prime}, 0, t\right)=0
$$


for any $y^{\prime} \in \mathbb{R}^{2}$ and $\left.s \in\right]-t_{k} M_{k}^{2}, 0[$. Condition (3.7) is replaced with

$$
\left|u^{(k)}\left(0, x_{3}^{(k)} M_{k}, 0\right)\right|=1 .
$$

Our aim is to understand what happens if $k \rightarrow \infty$.

Without loss of generality, we may assume that the following statements are true.

Scenario 1. There exists a divergence free function $u \in L_{\infty}\left(Q_{-}\right)$such that $|u| \leq 1$ a.e. in $Q_{-}$and, for any $R>0$,

$$
\int_{Q(R)} u^{(k)} \cdot w d x d t \rightarrow \int_{Q(R)} u \cdot w d x d t
$$

for any $w \in L_{1}(Q(R))$. Here, $\left.Q(R)=B(R) \times\right]-R^{2}, 0[$.

Scenario 2. There exists a divergence free function $u \in L_{\infty}\left(Q_{-}^{+}\right)$such that $|u| \leq 1$ a.e. in $Q_{-}^{+}$and, for any $R>0$,

$$
\int_{Q_{+}(R)} u^{(k)} \cdot w d x d t \rightarrow \int_{Q_{+}(R)} u \cdot w d x d t
$$

for any $w \in L_{1}\left(Q_{+}(R)\right)$. Here, $\left.Q_{+}(R)=B_{+}(R) \times\right]-R^{2}, 0[$.

\section{$\S 4$. SCEnARIO 1}

Our goal is to show that the limit function $u$ in (3.12) must be a mild bounded ancient solution in the entire space and satisfy the condition

$$
|u(0,0)|=1 .
$$

Fix an arbitrary $A<0$. Let $k$ be sufficiently large so that $A>-t_{k} M_{k}^{2}$. We split $w:=u^{(k)}$ into two parts

$$
w=w^{1}+w^{2}
$$

so that

$$
\partial_{t} w^{1}-\Delta w^{1}+\nabla r^{1}=0, \quad \operatorname{div} w^{1}=0
$$

in $\left.Q_{A}^{k}=\mathbb{R}^{2} \times\left\{y_{3}>-d_{k}\right\} \times\right] A, 0\left[\right.$, where $d_{k}=x_{3}^{(k)} M_{k} \rightarrow \infty$,

$$
w^{1}\left(y^{\prime},-d_{k}, t\right)=0
$$

for $\left.\left(y^{\prime}, t\right) \in \mathbb{R}^{2} \times\right] A, 0[$, and

$$
w^{1}(y, A)=w(y, A)
$$

for $y \in \mathbb{R}^{2} \times\left\{y_{3}>-d_{k}\right\}$.

The second part of $w$ is a solution to the following initial boundary value problem:

$$
\partial_{t} w^{2}-\Delta w^{2}+\nabla r^{2}=\operatorname{div} F+\nabla p^{1(k)}, \quad \operatorname{div} w^{2}=0
$$

in $Q_{A}^{k}$,

$$
w^{2}\left(y^{\prime},-d_{k}, t\right)=0
$$

for $\left.\left(y^{\prime}, t\right) \in \mathbb{R}^{2} \times\right] A, 0[$, and

$$
w^{2}(y, A)=0
$$

for $y \in \mathbb{R}^{2} \times\left\{y_{3}>-d_{k}\right\}$. Here, $F=w \otimes w$ and $p^{1(k)}$ is defined by the following Neumann boundary value problem

$$
\begin{aligned}
& \Delta p^{1(k)}(y, t)=-\operatorname{div} \operatorname{div} F(y, t), \quad(y, t) \in Q_{A}^{k}, \\
& \left.p_{, 3}^{1(k)}\left(y^{\prime},-d_{k}, t\right)=0 \quad\left(y^{\prime}, t\right) \in \mathbb{R}^{2} \times\right] A, 0[.
\end{aligned}
$$


Keeping (2.4) in mind, we can present $w^{1}$ in the form $w^{1}=w^{1,1}+w^{1,2}$, where

$$
w^{1,1}(y, t)=\int_{\mathbb{R}^{2} \times\left\{y_{3}>-d_{k}\right\}} G^{1}\left(y+d_{k} e_{3}, z+d_{k} e_{3}, t-A\right) w(z, A) d z
$$

and

$$
w^{1,2}(y, t)=\int_{\mathbb{R}^{2} \times\left\{y_{3}>-d_{k}\right\}} G^{2}\left(y+d_{k} e_{3}, z+d_{k} e_{3}, t-A\right) w(z, A) d z .
$$

Elementary calculations, estimate (2.8), and the inequality $|w(y, A)| \leq 1$ ensure an upper bound for $w^{1,2}$ :

$$
\left|w^{1,2}(y, t)\right| \leq c \frac{\sqrt{t-A}}{y_{3}+d_{k}}
$$

A similar decomposition can be exploited in order to evaluate $w^{2}$. We have $w^{2}(y, t)=$ $w^{2,1}+w^{2,2}$, where

$$
w^{2,1}(y, t)=-\int_{A}^{0} \int_{\mathbb{R}^{2} \times\left\{y_{3}>-d_{k}\right\}} G^{1}\left(y+d_{k} e_{3}, z+d_{k} e_{3}, t-\tau\right)\left(\operatorname{div} F+\nabla p^{1(k)}\right)(z, \tau) d z d \tau
$$

and

$w^{2,2}(y, t)=-\int_{A}^{0} \int_{\mathbb{R}^{2} \times\left\{y_{3}>-d_{k}\right\}} G^{2}\left(y+d_{k} e_{3}, z+d_{k} e_{3}, t-\tau\right)\left(\operatorname{div} F+\nabla p^{1(k)}\right)(z, \tau) d z d \tau$.

In fact, the velocity $w^{2,2}$ obeys the same estimate as (4.1)

$$
\left|w^{2,2}(y, t)\right| \leq c \frac{\sqrt{t-A}}{y_{3}+d_{k}}
$$

To prove (4.2), we need the following lemma.

Lemma 4.1. Let

$$
V_{i}(x, t)=\int_{0}^{t} \int_{\mathbb{R}_{+}^{3}} K_{i j m}^{2}(x, y, t-\tau) H_{k m}(y, \tau) d y d \tau
$$

with $K_{i j m}^{2}=\bar{K}_{i j m}^{2}+\widehat{K}_{i j m}^{2}$, where $\bar{K}_{i s m}^{2}(x, z, t)$ is a linear combination of the terms $\frac{\partial G_{i j}^{2}}{\partial y_{k}}(x, z, t)$ and $\hat{K}_{i s m}^{2}(x, z, t)$ is a linear combination of the terms

$$
\frac{\partial^{2}}{\partial x_{\alpha} \partial x_{\beta}} \int_{\mathbb{R}_{+}^{3}} G_{i j}^{2}(x, y, t) \frac{\partial N^{( \pm)}}{\partial y_{s}}(y, z) d y .
$$

Then $|V(x, t)| \leq c\|H\|_{\infty} \frac{t}{x_{3}}$.

Proof. By (2.8), we find

$$
\left|\bar{K}^{2}(x, y, t)\right| \leq \frac{c}{t^{\frac{1}{2}}\left(\left|x-y^{*}\right|^{2}+t\right)^{\frac{3}{2}}} \exp \left(-\frac{c y_{3}^{2}}{t}\right)
$$


For the second term $\widehat{K}^{2}$, we are going to make use of estimate (2.11). So, we have

$$
\begin{aligned}
& |V(x, t)| \leq c\|H\|_{\infty} \int_{0}^{t} \int_{\mathbb{R}_{+}^{3}}\left|K^{2}(x, z, t-\tau)\right| d z d \tau \\
& \quad \leq c\|H\|_{\infty} \int_{0}^{t} \int_{\mathbb{R}_{+}^{3}}\left(\frac{1}{\tau^{\frac{1}{2}}\left(\left|x-z^{*}\right|^{2}+\tau\right)^{\frac{3}{2}}} \exp \left(-\frac{c z_{3}^{2}}{\tau}\right)+\frac{1}{\left(\left|x-z^{*}\right|^{2}+\tau\right)^{2}}\right) d z d \tau \\
& \quad \leq c\|H\|_{\infty} \int_{0}^{t} \int_{0}^{\infty}\left(\frac{1}{\tau^{\frac{1}{2}}\left(\left|x_{3}+z_{3}\right|^{2}+\tau\right)^{\frac{1}{2}}} \exp \left(-\frac{c z_{3}^{2}}{\tau}\right)+\frac{1}{\left|x_{3}+z_{3}\right|^{2}+\tau}\right) d z_{3} d \tau \\
& \quad \leq c\|H\|_{\infty}\left(\frac{1}{x_{3}} \int_{0}^{t} \int_{0}^{\infty} \frac{1}{\tau^{\frac{1}{2}}} \exp \left(-\frac{c z_{3}^{2}}{\tau}\right) d z_{3} d \tau+\int_{0}^{t} \int_{x_{3}}^{\infty} \frac{1}{|u|^{2}+\tau} d u d \tau\right) \\
& \quad \leq c\|H\|_{\infty}\left(\frac{t}{x_{3}} \int_{0}^{\infty} \exp \left(-c u^{2}\right) d u+\int_{0}^{t} \frac{1}{\tau^{\frac{1}{2}}}\left(\frac{\pi}{2}-\arctan \left(\frac{x_{3}}{\tau^{\frac{1}{2}}}\right)\right) d \tau\right) .
\end{aligned}
$$

To estimate the last integral, we do the following:

$$
\int_{0}^{t} \frac{1}{\tau^{\frac{1}{2}}}\left(\frac{\pi}{2}-\arctan \left(\frac{x_{3}}{\tau^{\frac{1}{2}}}\right)\right) d \tau=\int_{0}^{t} \frac{1}{\tau^{\frac{1}{2}}} \arctan \left(\frac{\tau^{\frac{1}{2}}}{x_{3}}\right) d \tau \leq \frac{t}{x_{3}} .
$$

Lemma 4.1 is proved.

Now, the upper bound (4.2) follows from Lemma 4.1 and the identity

$$
w_{i}^{2,2}(y, t)=\int_{A}^{0} \int_{\mathbb{R}^{2} \times\left\{y_{3}>-d_{k}\right\}} K_{i j m}^{2}\left(y+d_{k} e_{3}, z+d_{k} e_{3}, t-\tau\right) F_{j m}(z, \tau) d z d \tau
$$

with the potential $K^{2}$ derived from the boundary value problem (2.7), in which $G$ is replaced with $G^{2}$.

If we let $w^{0}(y, t)=w^{1,1}+w^{2,1}$, then the new function is a solution of the following initial boundary value problem:

$$
\partial_{t} w^{0}-\Delta w^{0}=\operatorname{div} F+\nabla p^{1(k)}
$$

in $Q_{A}^{k}$,

for $\left.\left(y^{\prime}, t\right) \in \mathbb{R}^{2} \times\right] A, 0[$, and

$$
w^{0}\left(y^{\prime},-d_{k}, t\right)=0
$$

$$
w^{0}(y, A)=w(y, A)
$$

for $y \in \mathbb{R}^{2} \times\left\{y_{3}>-d_{k}\right\}$. Using exact representation formulas for $w^{1,1}$ and $w^{2,1}$, we may assume that $w^{0}$ is bounded by a constant $c$, which is independent of $k$. On the other hand, we know that the function $p^{1(k)}$, being extended to the whole $\mathbb{R}^{3}$ so that $p^{1(k)}\left(y^{\prime}, y_{3}, t\right)=$ $p^{1(k)}\left(y^{\prime}, y_{3}+2 d_{k}, t\right)$ for $y_{3}<-d_{k}$, belongs to the space $L_{\infty}\left(A, 0 ; B M O\left(\mathbb{R}^{3}\right)\right)$ and the corresponding norm is bounded by a constant independent of $k$. So, we have

$$
\sup _{A \leq t \leq 0} \int_{B(x, \sqrt{-A})}\left|p^{1(k)}(y, t)-\left[p^{1(k)}\right]_{B(x, \sqrt{-A})}(t)\right|^{m} d y \leq c(m)(-A)^{\frac{3}{2}} .
$$

This means, see Appendix II, that the sequence $w^{0}$ is precompact in $C(K \times[A / 2,0])$, where $K$ is an arbitrary compact subset of $\mathbb{R}^{3}$. Now, it remains to make use of estimates for $w^{1,2}$ and $w^{2,2}$, pass to the limit in the equation for $w^{0}$ taking into account that $F=w \otimes w$, and conclude that, by the arbitrariness of $A, u$ is a mild bounded ancient solution to the Navier-Stokes equations satisfying $|u(0,0)|=1$.

Remark 4.2. In fact, we have shown that

$$
u^{(k)} \rightarrow u
$$

uniformly on the closure of the set $Q(R)$ for any $R>0$. 


\section{$\S 5$. Scenario 2}

Here, we are going to prove the following statement.

Proposition 5.1. We have

$$
u^{(k)} \rightarrow u
$$

uniformly on the closure of the set $\left.Q_{+}(R)=B_{+}(R) \times\right]-R^{2}, 0\left[\right.$ with $B_{+}(R)=\{x \in B(R)$ : $\left.x_{3}>0\right\}$ for any $R>0$. The limit function $u$ is equal to zero at $y_{3}=0$ and is nontrivial in the sense that

$$
|u(0, a, 0)|=1
$$

with $a>0$.

Proof. As in the previous section, we split $w$ into two parts

$$
w=w^{1}+w^{2},
$$

where

$$
\partial_{t} w^{1}-\Delta w^{1}+\nabla r^{1}=0, \quad \operatorname{div} w^{1}=0
$$

in $\left.Q_{A}=\mathbb{R}_{+}^{3} \times\right] A, 0[$,

$$
w^{1}\left(y^{\prime}, 0, t\right)=0
$$

for $\left.\left(y^{\prime}, t\right) \in \mathbb{R}^{2} \times\right] A, 0[$, and

$$
w^{1}(y, A)=w(y, A)
$$

for $y \in \mathbb{R}_{+}^{3}$.

The second part is a solution of the following problem:

$$
\partial_{t} w^{2}-\Delta w^{2}+\nabla r^{2}=\operatorname{div} F+\nabla p^{1(k)}, \quad \operatorname{div} w^{2}=0
$$

in $Q_{A}$,

for $\left.\left(y^{\prime}, t\right) \in \mathbb{R}^{2} \times\right] A, 0[$, and

$$
w^{2}\left(y^{\prime}, 0, t\right)=0
$$

$$
w^{2}(y, A)=0
$$

for $y \in \mathbb{R}_{+}^{3}$. Here, $F=w \otimes w$ and $p^{1(k)}$ is defined by the following Neumann boundary value problem:

$$
\begin{aligned}
\Delta p^{1(k)}(y, t) & =-\operatorname{div} \operatorname{div} F(y, t), \quad(y, t) \in Q_{A}, \\
p_{, 3}^{1(k)}\left(y^{\prime}, 0, t\right) & \left.=0, \quad\left(y^{\prime}, t\right) \in \mathbb{R}^{2} \times\right] A, 0[.
\end{aligned}
$$

First, we discuss the precompactness of $w^{2}$ in $C\left(\bar{B}_{+}(R) \times[A / 2,0]\right)$ for any positive $R$. Indeed, by (2.8), we have

$$
\left|\frac{\partial G_{i j}^{2}}{\partial z_{i}}(x, z, t)\right| \leq \frac{c}{t^{\frac{1}{2}}\left(\left|x-z^{*}\right|^{2}+t\right)^{\frac{3}{2}}} \exp \left(-\frac{c z_{3}^{2}}{t}\right)
$$

and, using estimates (2.9) and the definition of the kernel $K$, it is not difficult to show that

$$
\int_{\mathbb{R}_{+}^{3}}\left|K_{i s m}(x, z, t)\right| d z d \tau \leq \frac{c}{\sqrt{t}} .
$$

Next, first, assuming $A \leq t_{1}<t_{2} \leq 0$ and $x^{1}, x^{2} \in \mathbb{R}_{+}^{3}$, we obtain

$$
\begin{aligned}
\left|w^{2}\left(x^{1}, t_{1}\right)-w^{2}\left(x^{2}, t_{2}\right)\right| & \leq\left|\int_{A}^{t_{1}} \int_{\mathbb{R}_{+}^{3}}\left(K\left(x^{1}, y, t_{1}-\tau\right)-K\left(x^{2}, y, t_{2}-\tau\right)\right) F(y, \tau) d y d \tau\right| \\
& +\left|\int_{t_{1}}^{t_{2}} \int_{\mathbb{R}_{+}^{3}} K\left(x^{2}, y, t_{2}-\tau\right) F(y, \tau) d y d \tau\right|=I_{1}+I_{2} .
\end{aligned}
$$


For the second term, we have

$$
I_{2} \leq c\|F\|_{\infty} \sqrt{t_{2}-t_{1}} .
$$

Next, in the first term, we make a change of variables:

$$
\begin{aligned}
I_{1} & \leq\|F\|_{\infty} \int_{0}^{t_{1}-A} \int_{\mathbb{R}_{+}^{3}}\left|K\left(x^{1}, y, \tau\right)-K\left(x^{2}, y, t_{2}-t_{1}+\tau\right)\right| d y d \tau \\
& \leq\|F\|_{\infty} \int_{0}^{-A} \int_{\mathbb{R}_{+}^{3}}\left|K\left(x^{1}, y, \tau\right)-K\left(x^{2}, y, t_{2}-t_{1}+\tau\right)\right| d y d \tau \\
& =\|F\|_{\infty} J\left(x^{1}, x^{2} ; t_{1}, t_{2}\right) .
\end{aligned}
$$

Using the above estimates of the Green function, it is not difficult to show that, given $\varepsilon>0$, there exists $\kappa(\varepsilon, A, R)$ such that if $\left|x^{1}-x^{2}\right|<\kappa, 0<t_{2}-t_{1}<\kappa, x^{1}, x^{2} \in \bar{B}_{+}(R)$, and $A \leq t_{1}<t_{2} \leq 0$, then $J\left(x^{1}, x^{2} ; t_{1}, t_{2}\right)<\varepsilon$. So, the required precompactness of $w^{2}$ follows from the Arzela-Ascoli theorem and from the inequality

$$
\left|w^{2}\left(x^{1}, t_{1}\right)-w^{2}\left(x^{2}, t_{2}\right)\right| \leq\|F\|_{\infty} J\left(x^{1}, x^{2} ; t_{1}, t_{2}\right)+c\|F\|_{\infty} \sqrt{t_{2}-t_{1}} .
$$

The precompactness of $w^{1}$ on the same sets is based on similar arguments and the following fact:

$$
\int_{\mathbb{R}_{+}^{3}}\left|G^{2}(x, y, t)\right| d y \leq c \sqrt{\frac{t}{x_{3}^{2}+t}} \leq c .
$$

So, as usual, applying the diagonal Cantor procedure, we select a subsequence still denoted by $u^{(k)}$ that converges uniformly on sets $\bar{Q}_{+}(n)$ for any natural $n$. On the other hand, $u^{(k)}\left(0, d_{k}, 0\right) \rightarrow u(0, a, 0)$ so that $|u(0, a, 0)|=1$. The latter actually implies that $a>0$.

The last arguments in the proof of Proposition 5.1 allow us to prove Proposition 1.5.

Proof of Proposition 1.5. If we assume that $T$ is a blow-up time, then, since $a \leq \varepsilon$, we have scenario 2 if one blows-up our solution $v$ and gets a function $u$ of Proposition 5.1. It is not so difficult to see that the modulus of continuity of $u$ in $\bar{Q}_{+}(2)$ depends only on the integral modulus of continuity of the above Green functions. So, there exists $\delta_{0}>0$ such that $|u(0,0,0)-u(0, a, 0)|<1 / 2$ provided $a \leq \min \left(1, \delta_{0}\right)$ but in fact $|u(0,0,0)-u(0, a, 0)|=|u(0, a, 0)|=1$. It remains to take $\varepsilon=\min \left(1, \delta_{0}\right)$.

Proposition 5.2. The limit function $u$ is a mild bounded ancient solution.

Proof. We have already proved that

$$
u^{(k)} \rightarrow u
$$

in $C\left(\bar{Q}_{+}(R)\right)$.

We define $p^{1(k)}$ as a solution of the following Neumann boundary value problem:

$$
\Delta p^{1(k)}(x, t)=-\operatorname{div} \operatorname{div}\left(u^{(k)} \otimes u^{(k)}\right)(x, t)
$$

for $(x, t) \in Q_{-}^{+}$and

$$
p_{, 3}^{1(k)}\left(x^{\prime}, 0, t\right)=0
$$

for $\left.\left(x^{\prime}, t\right) \in \mathbb{R}^{2} \times\right]-\infty, 0\left[\right.$. We extend $p^{1(k)}$ to the entire space $\mathbb{R}^{3}$ in the even way with respect to $x_{3}$, and $u^{(k)}$ is assumed to be extended by zero. Then $H^{(k)}:=u^{(k)} \otimes u^{(k)}+$ $\mathbb{I} p^{1(k)} \in L_{\infty}\left(-\infty, 0 ; B M O\left(\mathbb{R}^{3}\right)\right)$, so that

$$
\sup _{k}\left(\left\|H^{(k)}\right\|_{L_{\infty}(B M O)}+\left\|u^{(k)}\right\|_{\infty}\right)=d<\infty .
$$


We know that

$$
u^{(k)}=\int_{\mathbb{R}_{+}^{3}} G(x, y, t-A) u^{(k)}(y, A) d y+\int_{A}^{t} \int_{\mathbb{R}_{+}^{3}} K(x, y, t-\tau) F^{(k)}(y, \tau) d y d \tau,
$$

where $F^{(k)}=u^{(k)} \otimes u^{(k)}$. All the norms, being bounded by $\left\|u^{(k)}\right\|_{\infty},\left\|F^{(k)}\right\|_{\infty}$, or $\left\|H^{(k)}\right\|_{L_{\infty}(B M O)}$ only, remain to be bounded for the limit functions.

We may transform the above formula by integration by parts in the second term on the right hand side:

$$
\begin{aligned}
u_{i}^{(k)}(x, t) & =\int_{\mathbb{R}_{+}^{3}} G_{i j}(x, y, t-A) u_{j}^{(k)}(y, A) d y \\
& +\int_{A}^{t} \int_{\mathbb{R}_{+}^{3}} \frac{\partial G_{i j}}{\partial y_{l}}(x, y, t-\tau)\left(H_{j l}^{(k)}(y, \tau)-\left[H_{j l}^{(k)}\right]_{B\left(\left(x^{\prime}, 0\right), a\right)}(\tau)\right) d y d \tau,
\end{aligned}
$$

where $B\left(\left(x^{\prime}, 0\right), a\right)$ is a ball of radius $a=\left(x_{3}^{2}+t-\tau\right)^{\frac{1}{2}}$ centered at the point $\left(x^{\prime}, 0\right)$.

Then we split $u^{(k)}=u_{(k)}^{1}+u_{(k)}^{2}$ according to the decomposition of the Green function: $G=G^{1}+G^{2}$, see (2.4). Next, let $u_{(k)}^{2}=u_{(k)}^{2,1}+u_{(k)}^{2,2}$ so that

$$
u_{i(k)}^{2,2}(x, t)=\int_{A}^{t} \int_{\mathbb{R}_{+}^{3}} \frac{\partial G_{i j}^{2}}{\partial y_{l}}(x, y, t-\tau)\left(H_{j l}^{(k)}(y, \tau)-\left[H_{j l}^{(k)}\right]_{B\left(\left(x^{\prime}, 0\right), a\right)}(\tau)\right) d y d \tau .
$$

From (2.8), it follows that

$$
\begin{aligned}
\left|\frac{\partial^{|\alpha|+|\gamma|} G^{2}}{\partial x^{\alpha} \partial y^{\gamma}}(x, y, t-A)\right| & \leq c(\alpha, \gamma)(t-A)^{-\frac{|\gamma|}{2}}\left(t-A+x_{3}^{2}\right)^{-\frac{|\alpha|}{2}} \\
& \times \exp \left(-\frac{c y_{3}^{2}}{t-A}\right)\left(\left|x^{\prime}-y^{\prime}\right|^{2}+y_{3}^{2}+x_{3}^{2}+t-A\right)^{-\frac{3}{2}} .
\end{aligned}
$$

Now, we have

$$
\begin{aligned}
\left|\nabla^{|\alpha|} u_{(k)}^{2,1}(x, t)\right| \leq & c(\alpha)\left(t-A+x_{3}^{2}\right)^{-\frac{|\alpha|}{2}} \\
& \times \int_{\mathbb{R}_{+}^{3}}\left(\left|x^{\prime}-y^{\prime}\right|^{2}+y_{3}^{2}+x_{3}^{2}+t-A\right)^{-\frac{3}{2}} \exp \left(-\frac{c y_{3}^{2}}{t-A}\right)\left|u^{(k)}(y, A)\right| d y
\end{aligned}
$$

and

$$
\begin{aligned}
& \left|\nabla^{|\alpha|} u_{(k)}^{2,2}(x, t)\right| \leq c(\alpha) \int_{A}^{t}(t-\tau)^{-\frac{1}{2}}\left(t-\tau+x_{3}^{2}\right)^{-\frac{|\alpha|}{2}} \\
& \times \int_{\mathbb{R}_{+}^{3}}\left(\left|x^{\prime}-y^{\prime}\right|^{2}+y_{3}^{2}+x_{3}^{2}+t-\tau\right)^{-\frac{3}{2}} \exp \left(-\frac{c y_{3}^{2}}{t-\tau}\right)\left|H^{(k)}(y, \tau)-\left[H^{(k)}\right]_{B\left(\left(x^{\prime}, 0\right), a\right)}(\tau)\right| d y d \tau .
\end{aligned}
$$

So, we find

$$
\left|\nabla^{|\alpha|} u_{(k)}^{2,1}(x, t)\right| \leq c(\alpha) d\left(x_{3}^{2}+t-A\right)^{-\frac{|\alpha|}{2}} \leq c(\alpha) d\left(x_{3}^{2}-A\right)^{-\frac{|\alpha|}{2}}
$$

for all $\left.(x, t) \in \mathbb{R}_{+}^{3} \times\right] A / 2,0\left[\right.$ and, by Lemma 6.1 with $\beta=\frac{t-\tau}{c}$ and $a^{2}=x_{3}^{2}+t-\tau$,

$$
\begin{aligned}
\left|\nabla^{|\alpha|} u_{(k)}^{2,2}(x, t)\right| & \leq c(\alpha) d \int_{A}^{t}(t-\tau)^{-\frac{1}{2}}\left(x_{3}^{2}+t-\tau\right)^{-\frac{|\alpha|}{2}} d \tau \\
& \leq c(\alpha) d \int_{0}^{\sqrt{t-A}} \frac{d q}{\left(x_{3}^{2}+q^{2}\right)^{\frac{|\alpha|}{2}}} \leq c(\alpha) d \int_{0}^{\sqrt{-A}} \frac{d q}{\left(x_{3}^{2}+q^{2}\right)^{\frac{|\alpha|}{2}}}
\end{aligned}
$$

for all $\left.(x, t) \in \mathbb{R}_{+}^{3} \times\right] A, 0[$. 
Next, we observe that $u_{(k)}^{1}$ is a solution of the following initial boundary value problem:

$$
\partial_{t} u_{(k)}^{1}-\Delta u_{(k)}^{1}=-\operatorname{div} H^{(k)}
$$

for any $\left.(x, t) \in \mathbb{R}_{+}^{3} \times\right] A, 0[$,

$$
u_{(k)}^{1}\left(x^{\prime}, 0, t\right)=0
$$

for any $\left(x^{\prime}, t\right)$ from $\left.\mathbb{R}^{2} \times\right] A, 0[$,

$$
u_{(k)}^{1}(x, A)=u^{(k)}(x, A)
$$

for all $x \in \mathbb{R}_{+}^{3}$.

Regarding $u_{(k)}^{2,2}(x, t)$, we can say the following: for an appropriate extension of it to the whole $\mathbb{R}^{3}$, we can claim that $\nabla u_{(k)}^{2,2} \in L_{\infty}(A, 0 ; B M O)$ and $\nabla u_{(k)}^{2,2} \in L_{\infty}\left(A, 0 ; L_{q, \text { unif }}\left(\mathbb{R}^{3}\right)\right)$ for any finite $q \geq 1$.

Since $H^{(k)}$ is bounded in $L_{\infty}\left(A, 0 ; B M O\left(\mathbb{R}^{3}\right)\right)$, by estimates (5.3) and (15.4), we have (from the energy inequality)

$$
\sup _{k} \int_{A / 2}^{0} \int_{\mathbb{R}_{+}^{3}} \varphi^{2}\left|\nabla u_{(k)}^{1}\right|^{2} d x d t<\infty
$$

for any test function $\varphi \in C_{0}^{\infty}\left(\mathbb{R}^{3} \times\right]-A / 2, A / 2[)$. Then, from the equation for the pressure $p^{1(k)}$, we derive a similar estimate for the gradient of $p^{1(k)}$ :

$$
\sup _{k} \int_{A / 2}^{0} \int_{\mathbb{R}_{+}^{3}} \varphi^{2}\left|\nabla p^{1(k)}\right|^{2} d x d t<\infty
$$

for any test function $\varphi \in C_{0}^{\infty}\left(\mathbb{R}^{3} \times\right]-A / 2, A / 2[)$. Using coercive estimates for the heat equation, we then get

$$
\sup _{k} \int_{A / 2}^{0} \int_{\mathbb{R}_{+}^{3}} \varphi^{2}\left(\left|\partial_{t} u_{(k)}^{1}\right|^{2}+\left|\nabla^{2} u_{(k)}^{1}\right|^{2}\right) d x d t<\infty
$$

for any test function $\varphi \in C_{0}^{\infty}\left(\mathbb{R}^{3} \times\right]-A / 2, A / 2[)$.

Next, we may use a parabolic embedding theorem to show that

$$
\sup _{k} \int_{A / 2}^{0} \int_{\mathbb{R}_{+}^{3}} \varphi^{2}\left|\nabla u_{(k)}^{1}\right|^{3} d x d t<\infty
$$

for the same test function $\varphi$. An estimate of the same type is valid for the gradient of $p^{1(k)}$. Repeating these arguments several times, we can state that $\nabla u_{(k)}^{1}$ is bounded in the domains $\left.\mathbb{R}_{+}^{3} \times\right] 3 A / 8,0\left[\right.$. The boundedness of $\nabla p^{1(k)}$ follows from local regularity and the equation $\Delta p^{1(k)}=-u_{i, j}^{(k)} u_{j, i}^{(k)}$, which is valid in $\mathbb{R}^{3}$ for appropriate extensions of $p^{1(k)}$ and $u_{i, j}^{(k)} u_{j, i}^{(k)}$ from $\mathbb{R}_{+}^{3}$ to $\mathbb{R}^{3}$. Summarizing all these estimates, taking the arbitrariness of $A$ into account, and using a shift in time, we see that

$$
\sup _{k}\left|\nabla u^{(k)}(x, t)\right| \leq c \ln \left(2+x_{3}^{-1}\right)
$$

for $(x, t) \in Q_{-}^{+}$, and

$$
\sup _{k} \sup _{(x, t) \in Q_{-}^{+}}\left|\nabla p^{1(k)}(x, t)\right| \leq C<\infty
$$


Now, we can go back to evaluation of the function $u_{(k)}^{2}$. By (5.11) and (5.12), we do not need integrating by parts in the expression for $u_{(k)}^{2,2}$ any more, so that

$$
u_{i(k)}^{2,2}(x, t)=-\int_{A}^{t} \int_{\mathbb{R}_{+}^{3}} G_{i j}^{2}(x, y, t-\tau)\left[\frac{\partial}{\partial y_{l}} F_{j l}^{(k)}(y, \tau)+\frac{\partial}{\partial y_{j}} p^{1(k)}(y, \tau)\right] d y d \tau .
$$

Next, by (5.11) and (5.12) and by estimates of the Green function $G^{2}$, we find the following bound:

$$
\begin{aligned}
& \left|\nabla u_{(k)}^{2,2}(x, t)\right| \leq c \int_{A}^{t} \frac{d \tau}{\left(x_{3}^{2}+t-\tau\right)^{\frac{1}{2}}} \int_{0}^{\infty} \frac{\exp \left(-\frac{c y_{3}^{2}}{t-\tau}\right)}{\left(x_{3}^{2}+y_{3}^{2}+t-\tau\right)^{\frac{1}{2}}} \ln \left(2+1 / y_{3}\right) d y_{3} \\
& \quad \leq c \int_{A}^{t} \frac{d \tau}{\left(x_{3}^{2}+t-\tau\right)^{\frac{3}{4}}} \int_{0}^{\infty} \frac{\exp \left(-\frac{c y_{3}^{2}}{t-\tau}\right)}{\left(x_{3}^{2}+y_{3}^{2}+t-\tau\right)^{\frac{1}{4}}} \ln \left(2+1 / y_{3}\right) d y_{3} \\
& \quad \leq c \int_{A}^{t} \frac{d \tau}{\left(x_{3}^{2}+t-\tau\right)^{\frac{3}{4}}}\left[\int_{0}^{1} y_{3}^{-\frac{1}{2}} \ln \left(2+1 / y_{3}\right) d y_{3}+\int_{1}^{\infty} \exp \left(-\frac{c y_{3}^{2}}{t-\tau}\right) d y_{3}\right] \\
& \quad \leq c(1+\sqrt{-A})(-A)^{\frac{1}{4}} .
\end{aligned}
$$

This implies that, in fact,

$$
\sup _{k} \sup _{(x, t) \in Q_{-}^{+}}\left|\nabla u^{(k)}(x, t)\right| \leq C<\infty .
$$

Using estimates (2.8), (5.2), (5.12), and bootstrap arguments, we show that

$$
\sup _{(x, t) \in Q_{-}^{+}, x_{3} \geq \delta}\left|\nabla^{l} u^{(k)}(x, t)\right|+\left|\nabla^{l+1} p^{1(k)}(x, t)\right| \leq C(l, \delta)<\infty
$$

for any $l \geq 0$ and for any $\delta>0$.

As to the derivatives in $t$, by (2.10), we find

$$
\begin{aligned}
& \left|\partial_{t} u_{(k)}^{2,2}(x, t)\right| \\
& \leq c \int_{A}^{t} \int_{0}^{\infty} \int_{\mathbb{R}^{2}} \frac{1}{t-\tau} \frac{1}{\left(\left|x^{\prime}-y^{\prime}\right|^{2}+x_{3}^{2}+y_{3}^{2}+t-\tau\right)^{\frac{3}{2}}} \exp \left(-\frac{c y_{3}^{2}}{t-\tau}\right)\left|\operatorname{div} H^{(k)}(y, \tau)\right| d y d \tau \\
& \leq c \int_{A}^{t} \frac{1}{(t-\tau)^{\frac{1}{2}}} \frac{1}{\left(x_{3}^{2}+t-\tau\right)^{\frac{1}{2}}} d \tau .
\end{aligned}
$$

So,

$$
\sup _{x^{\prime} \in \mathbb{R}^{2}}\left|\partial_{t} u_{(k)}^{2,2}\left(x^{\prime}, x_{3}, t\right)\right| \leq c \int_{0}^{t-A} \frac{1}{\vartheta^{\frac{1}{2}}} \frac{1}{\left(x_{3}^{2}+\vartheta\right)^{\frac{1}{2}}} d \vartheta .
$$

For the first term, we show in the same way that

$$
\left|\partial_{t} u_{(k)}^{2,1}(x, t)\right| \leq c \int_{\mathbb{R}_{+}^{3}}\left|\partial_{t} G^{2}(x, y, t-A)\right|\left|u^{(k)}(y, A)\right| d y
$$

and thus

$$
\left|\partial_{t} u_{(k)}^{2,1}(x, t)\right| \leq \frac{1}{(t-A)^{\frac{1}{2}}} \frac{1}{\left(x_{3}^{2}+t-A\right)^{\frac{1}{2}}} .
$$

From equations (5.5), (5.15), and (5.16), we can deduce that

$$
\sup _{(x, t) \in Q_{-}^{+}, x_{3} \geq \delta}\left|\partial_{t} u^{(k)}(x, t)\right| \leq c(\delta) .
$$


After passing to the limit as $k \rightarrow \infty$, we see that $u$ satisfies the standard integral identity with divergence free test functions. Then one can claim that there exists $p^{2}$ such that

$$
\partial_{t} u-\Delta u+\nabla p^{2}=-\operatorname{div} H
$$

in $Q_{-}^{+}$.

Obviously, $p^{2}(\cdot, t)$ is a harmonic function in a half-space whose gradient is bounded in $t$, in $x^{\prime}$, and in $x_{3} \geq \delta$ for any $\delta>0$. Taking the limit in (5.5), we show that $\nabla p^{2}$ satisfies

$$
\partial_{t} u^{2}-\Delta u^{2}+\nabla p^{2}=0
$$

in $\left.\mathbb{R}_{+}^{3} \times\right] A, 0[$. This, together with (5.3), (5.4) and (5.15), (5.16), implies that

$$
\sup _{x^{\prime} \in \mathbb{R}^{2}}\left|\nabla p^{2}\left(x^{\prime}, x_{3}, t\right)\right| \rightarrow 0
$$

as $x_{3} \rightarrow \infty$ for each $-\infty<t<0$. Moreover, a more detailed analysis of the above estimates for Green functions allows us to state that

$$
\left|\nabla p^{2}(x, t)\right| \leq c \ln \left(2+1 / x_{3}\right)
$$

for all $(x, t) \in Q_{-}^{+}$. Proposition 5.2 is proved.

Proof of Theorem 1.4 easily follows from the above arguments.

\section{$\S 6$. Appendix I}

Lemma 6.1. Assume that numbers $m$ and $\alpha_{0}$ satisfy the condition

$$
0<\alpha_{0}<\frac{m-1}{3}
$$

Let $a$ and $\beta$ be positive numbers, and let

$$
I(a, \beta):=\int_{\mathbb{R}^{3}} \frac{\left|f(x)-[f]_{B(a)}\right|}{\left(|x|^{2}+a^{2}\right)^{\frac{3}{2}}} \exp \left(-\frac{x_{3}^{2}}{\beta}\right) d x,
$$

where $[f]_{B(R)}$ is the mean value of $f$ over the ball $B(R)$ of radius $R$ centered at the origin. Let further $n=\frac{m}{m-1}, m_{1}=\frac{m}{1+\alpha_{0}}$, and $n_{1}=\frac{m_{1}}{m_{1}-1}$. Then

$$
\begin{aligned}
|I(a, \beta)| & \leq c\left(m, \alpha_{0}\right)\|f\|_{B M O\left(\mathbb{R}^{3}\right)} a^{-\frac{3 \alpha_{0}}{m}} \\
& \times\left(\sqrt{\beta} \int_{0}^{\infty} \exp \left(-u^{2}\right) \frac{d u}{\left(u^{2} \beta n_{1} / n+a^{2}\right)^{\frac{3 n}{2 n_{1}}-1}}\right)^{\frac{1}{n}} .
\end{aligned}
$$

Proof. Without loss of generality, we may assume that $[f]_{B(a)}=0$. We also put

$$
K(x):=\frac{1}{\left(|x|^{2}+a^{2}\right)^{\frac{3}{2}}} \exp \left(-\frac{x_{3}^{2}}{\beta}\right), \quad A:=\|f\|_{B M O\left(\mathbb{R}^{3}\right)} .
$$

Then, by the Hölder inequality,

$$
\begin{aligned}
|I(a, \beta)| & \leq \int_{\mathbb{R}^{3}} K^{\frac{1}{m_{1}}+\frac{1}{n_{1}}}(x)|f(x)| d x \\
& \leq\left(\int_{\mathbb{R}^{3}} K^{\frac{m}{m_{1}}}(x)|f(x)|^{m} d x\right)^{\frac{1}{m}}\left(\int_{\mathbb{R}^{3}} K^{\frac{n}{n_{1}}}(x) d x\right)^{\frac{1}{n}} .
\end{aligned}
$$

For the second factor on the right-hand side of the last inequality, we find

$$
\begin{aligned}
\int_{\mathbb{R}^{3}} K^{\frac{n}{n_{1}}}(x) d x & =\int_{-\infty}^{\infty} \exp \left(-\frac{x_{3}^{2} n}{\beta n_{1}}\right) d x_{3} 2 \pi \int_{0}^{\infty} \frac{\varrho d \varrho}{\left(\varrho^{2}+x_{3}^{2}+a^{2}\right)^{\frac{3 n}{2 n_{1}}}} \\
& =2 \pi \frac{1}{\frac{3 n}{2 n_{1}}-1} \int_{0}^{\infty} \exp \left(-\frac{x_{3}^{2} n}{\beta n_{1}}\right) d x_{3} \frac{1}{\left(x_{3}^{2}+a^{2}\right)^{\frac{3 n}{2 n_{1}}-1}} .
\end{aligned}
$$


Here, we have used condition (6.1) that ensures the inequality $\frac{3 n}{2 n_{1}}-1>0$. After changing variables, we have

$$
\left(\int_{\mathbb{R}^{3}} K^{\frac{n}{n_{1}}}(x) d x\right)^{\frac{1}{n}} \leq c(m, \alpha)\left(\sqrt{\beta} \int_{0}^{\infty} \exp \left(-u^{2}\right) \frac{d u}{\left(u^{2} \beta n_{1} / n+a^{2}\right)^{\frac{3 n}{2 n_{1}}-1}}\right)^{\frac{1}{n}} .
$$

Next,

$$
\begin{aligned}
\int_{\mathbb{R}^{3}} K^{\frac{m}{m_{1}}}(x)|f(x)|^{m} d x=\int_{B(a)} & K^{\frac{m}{m_{1}}}(x)|f(x)|^{m} d x \\
& +\sum_{k=0}^{\infty} \int_{B\left(a 2^{k+1}\right) \backslash B\left(a 2^{k}\right)} K^{\frac{m}{m_{1}}}(x)|f(x)|^{m} d x=I_{1}+I_{2} .
\end{aligned}
$$

Obviously,

$$
I_{1} \leq c(m) A^{m} a^{3-\frac{3 m}{m_{1}}} .
$$

For $I_{2}$, we are going to use the fact that $m / m_{1}=1+\alpha_{0}$ and the inequalities

$$
|f|^{m} \leq 2^{m-1}\left(\left|f-[f]_{B\left(a 2^{k+1}\right)}\right|^{m}+\left|[f]_{B\left(a 2^{k+1}\right)}\right|^{m}\right)
$$

and

$$
\left|[f]_{B\left(a 2^{k+1}\right)}\right| \leq c(k+1) A
$$

The latter inequality can be found for example in [16, p. 141]. Then, we have

$$
\begin{gathered}
I_{2} \leq c(m) \sum_{k=0}^{\infty} \frac{\left|B\left(a 2^{k+1}\right)\right|}{\left(a 2^{k}\right)^{3\left(1+\alpha_{0}\right)}} \frac{1}{\left|B\left(a 2^{k+1}\right)\right|} \int_{B\left(a 2^{k+1}\right)}\left|f-[f]_{B\left(a 2^{k+1}\right)}\right|^{m} \\
\quad+c(m) \sum_{k=0}^{\infty} \frac{\left|B\left(2^{k+1}\right)\right|}{\left(a 2^{k}\right)^{3\left(1+\alpha_{0}\right)}\left|[f]_{B\left(a 2^{k+1}\right)}\right|^{m}} \\
\leq c(m) A^{m} \sum_{k=0}^{\infty} \frac{1}{\left(a 2^{k}\right)^{3 \alpha_{0}}}(k+1)^{m} \leq c\left(m, \alpha_{0}\right) a^{-3 \alpha_{0}} A^{m} .
\end{gathered}
$$

This completes the proof of Lemma 6.1.

\section{$\S 7$. Appendix II}

Consider the following problem:

$$
\partial_{t} u-\Delta u=-\operatorname{div} g
$$

in $\mathcal{Q}(a)=Q(a)+\left(0, a^{2}\right)$. Assuming that $u$ and $g$ are sufficiently smooth in $\mathcal{Q}(a)$, we wish to estimate the modulus of continuity of $u$ in the closure of the set $B(a / 2) \times] 3(a / 2)^{2}, a^{2}[$ in terms of $\|u\|_{\infty, \mathcal{Q}(a)}$ and $\left\|g-[g]_{B(a)}\right\|_{L_{m, \infty}(\mathcal{Q}(a))}$ for sufficiently large $m \geq 1$.

First, we take a positive cut-off function $\varphi \in C_{0}^{\infty}(B(a) \times] 0,2 a^{2}[)$ and extend it by zero. We let $w=\varphi u$. This function solves the following Cauchy problem:

$$
\partial_{t} w-\Delta w=-\operatorname{div}(\varphi f)+f \nabla \varphi+w\left(\partial_{t} \varphi+\Delta \varphi\right)-2 \operatorname{div}(u \nabla \varphi)
$$

in $\left.\mathbb{R}^{3} \times\right] 0, a^{2}\left[\right.$, where $f(x, t)=g(x, t)-[g]_{B(a)}(t)$, and $w(x, 0)=0$ for $x \in \mathbb{R}^{3}$.

We split $w$ into two parts $w=w^{1}+w^{2}$, where

$$
\partial_{t} w^{1}-\Delta w^{1}=-\operatorname{div}(\varphi f)-2 \operatorname{div}(u \nabla \varphi)
$$

in $\left.\mathbb{R}^{3} \times\right] 0, a^{2}\left[\right.$ with $w^{1}(\cdot, 0)=0$ in $\mathbb{R}^{3}$, and

$$
\partial_{t} w^{2}-\Delta w^{2}=f \nabla \varphi+w\left(\partial_{t} \varphi+\Delta \varphi\right)
$$


in $\left.\mathbb{R}^{3} \times\right] 0, a^{2}\left[\right.$ with $w^{2}(\cdot, 0)=0$ in $\mathbb{R}^{3}$. The second part can be estimated with the help of coercive estimates:

$$
\left\|w^{2}\right\|_{W_{m}^{2,1}\left(\mathbb{R}^{3} \times\right] 0, a^{2}[)} \leq C\left(a, m,\|u\|_{\infty, \mathcal{Q}(a)},\|f\|_{L_{m, \infty}(\mathcal{Q}(a))}\right) .
$$

For sufficiently large $m$, they give an estimate for a Hölder norm of $u$; thus, we get a required estimate for the modulus of continuity of $w^{2}$.

For the first part, we make use of the solution formula

$$
\begin{aligned}
w^{1}(x, t) & \left.=-\int_{0}^{t} \int_{\mathbb{R}^{3}} \Gamma(x-y, t-\tau)(\operatorname{div}(\varphi f)(y, \tau)+2 \operatorname{div}(u \nabla \varphi))(y, \tau)\right) d y d \tau \\
& \left.=-\int_{0}^{t} \int_{\mathbb{R}^{3}} \nabla_{x} \Gamma(x-y, t-\tau) \cdot((\varphi f)(y, \tau)+2(u \nabla \varphi))(y, \tau)\right) d y d \tau .
\end{aligned}
$$

We first assume that $m>2012$ and observe that the following simple estimate is true:

$$
\int_{\mathbb{R}^{3}}|K(x-y, t-\tau)| d y \leq \frac{c}{\sqrt{t-\tau}},
$$

where $K(x-y, t)=\nabla_{x} \Gamma(x-y, t)$. Now, we wish to show that, for a given positive $\varepsilon$, there exists a positive $\delta(m, a)$ such that if $\left.\left(x^{1}, t_{1}\right),\left(x^{2}, t_{2}\right) \in B(a) \times\right] 0, a^{2}\left[\right.$ with $t_{2}>t_{1}$ and $\left|x^{1}-x^{2}\right|+t_{2}-t_{1}<\delta$, then

$$
\int_{0}^{a^{2}}\left(\int_{\mathbb{R}^{3}}\left|K\left(x^{2}-y, t_{2}-t_{1}+\vartheta\right)-K\left(x^{1}-y, \vartheta\right)\right| d y\right)^{\frac{1}{m^{\prime}}} \frac{1}{\vartheta^{\frac{2}{m}}} d \vartheta<\varepsilon .
$$

Assume that this is false. Then there exists $\varepsilon_{0}>0$ and sequences

$$
\left.\left(x^{1, n}, t_{1, n}\right),\left(x^{2, n}, t_{2, n}\right) \in B(a) \times\right] 0, a^{2}[
$$

such that $t_{2, n}>t_{1, n}$ and $\left|x^{1, n}-x^{2, n}\right|+t_{2, n}-t_{1, n} \rightarrow 0$, but

$$
\int_{0}^{a^{2}}\left(\int_{\mathbb{R}^{3}}\left|K\left(x^{2, n}-y, t_{2, n}-t_{1, n}+\vartheta\right)-K\left(x^{1, n}-y, \vartheta\right)\right| d y\right)^{\frac{1}{m^{\prime}}} \frac{1}{\vartheta \frac{2}{m}} d \vartheta \geq \varepsilon_{0} .
$$

Indeed, for any positive $\vartheta$,

$$
\int_{\mathbb{R}^{3}}\left|K\left(x^{2, n}-y, t_{2, n}-t_{1, n}+\vartheta\right)-K\left(x^{1, n}-y, \vartheta\right)\right| d y \rightarrow 0
$$

by the Lebesgue theorem. But then

$$
\int_{0}^{a^{2}}\left(\int_{\mathbb{R}^{3}}\left|K\left(x^{2, n}-y, t_{2, n}-t_{1, n}+\vartheta\right)-K\left(x^{1, n}-y, \vartheta\right)\right| d y\right)^{\frac{1}{m^{\prime}}} \frac{1}{\vartheta^{\frac{2}{m}}} d \vartheta \rightarrow 0,
$$

because

$$
\left(\int_{\mathbb{R}^{3}}\left|K\left(x^{2, n}-y, t_{2, n}-t_{1, n}+\vartheta\right)-K\left(x^{1, n}-y, \vartheta\right)\right| d y\right)^{\frac{1}{m^{\prime}}} \frac{1}{\vartheta^{\frac{2}{m}}} \leq \frac{c}{\vartheta^{\frac{1}{2 m^{\prime}}}} \frac{1}{\vartheta^{\frac{2}{m}}} \leq \frac{c}{\vartheta^{\frac{1}{2}+\frac{3}{2 m}}} .
$$

Since the function on the right-hand side is integrable under our assumption on $m$, we arrive at a contradiction again, by the Lebesgue dominated convergence theorem. The rest goes as follows:

$$
\begin{aligned}
& \left|w^{1}\left(x^{2}, t_{2}\right)-w^{1}\left(x^{1}, t_{1}\right)\right| \\
& \left.\leq \mid \int_{t_{1}}^{t_{2}} \int_{\mathbb{R}^{3}} K\left(x_{2}-y, t_{2}-\tau\right) \cdot((\varphi f)(y, \tau)+2(u \nabla \varphi))(y, \tau)\right) d y d \tau \mid \\
& \left.+\mid \int_{0}^{t_{1}} \int_{\mathbb{R}^{3}}\left(K\left(x_{2}-y, t_{2}-\tau\right)-K\left(x_{1}, t_{1}-\tau\right)\right)((\varphi f)(y, \tau)+2(u \nabla \varphi))(y, \tau)\right) d y d \tau \mid .
\end{aligned}
$$


Then we apply the Hölder inequality to obtain

$$
\begin{aligned}
& \left|w^{1}\left(x^{2}, t_{2}\right)-w^{1}\left(x^{1}, t_{1}\right)\right| \leq \int_{t_{1}}^{t_{2}}\left(\int_{\mathbb{R}^{3}}\left|K\left(x_{2}-y, t_{2}-\tau\right)\right| d y\right)^{\frac{1}{m^{\prime}}} \\
& \times\left(\int_{B(a)}\left|K\left(x_{2}-y, t_{2}-\tau\right)\right|\left(|f|^{m}+|u|^{m}\right) d y d \tau\right)^{\frac{1}{m}} \\
& +\int_{0}^{a^{2}}\left(\int_{\mathbb{R}^{3}}\left|K\left(x_{2}-y, t_{2}-\tau\right)-K\left(x_{1}, t_{1}-\tau\right)\right| d y\right)^{\frac{1}{m^{\prime}}} \\
& \times\left(\int_{B(a)}\left|K\left(x_{2}-y, t_{2}-\tau\right)-K\left(x_{1}, t_{1}-\tau\right)\right|\left(|f|^{m}+|u|^{m}\right) d y d \tau\right)^{\frac{1}{m}} \\
& \leq C\left(a, m,\|u\|_{\infty, \mathcal{Q}(a)},\|f\|_{L_{m, \infty}(\mathcal{Q}(a))}\right)\left(\int_{t_{1}}^{t_{2}} \frac{1}{\left(t_{2}-\tau\right)^{\frac{1}{2}+\frac{3}{2 m}}} d \tau\right. \\
& \left.+\int_{0}^{a^{2}}\left(\int_{\mathbb{R}^{3}}\left|K\left(x^{2}-y, t_{2}-t_{1}+\vartheta\right)-K\left(x^{1}-y, \vartheta\right)\right| d y\right)^{\frac{1}{m^{\prime}}} \frac{1}{\vartheta^{\frac{2}{m}}} d \vartheta\right) \\
& =C\left(a, m,\|u\|_{\infty, \mathcal{Q}(a)},\|f\|_{L_{m, \infty}(\mathcal{Q}(a))}\right)\left(\left(t_{2}-t_{1}\right)^{\frac{1}{2}-\frac{3}{2 m}} d\right. \\
& \left.+\int_{0}^{a^{2}}\left(\int_{\mathbb{R}^{3}}\left|K\left(x^{2}-y, t_{2}-t_{1}+\vartheta\right)-K\left(x^{1}-y, \vartheta\right)\right| d y\right)^{\frac{1}{m^{\prime}}} \frac{1}{\vartheta^{\frac{2}{m}}} d \vartheta\right) .
\end{aligned}
$$

Together with the above statements, this gives a required estimate for the modulus of continuity in the closure of $B(a / 2) \times] 3(a / 2)^{2}, a^{2}[$.

\section{REFERENCES}

[1] L. Caffarelli, R.-V. Kohn, and L. Nirenberg, Partial regularity of suitable weak solutions of the Navier-Stokes equations, Comm. Pure Appl. Math. 35 (1982), no. 6, 771-831. MR673830 (84m:35097)

[2] J. G. Heywood, The Navier-Stokes equations: on the existence, regularity and decay of solutions, Indiana Univ. Math. J. 29 (1980), no. 5, 639-681. MR.589434 (81k:35131)

[3] H. Jia, G. A. Seregin, and V. Śverák, Liouville theorems in unbounded domains for the timedependent Stokes system, J. Math. Phys. 53 (2012), no. 11, 115604. MR3026549

[4] _ A Liouville theorem for the Stokes system in half-space, Zap. Nauchn. Sem. S.-Peterburg. Otdel. Mat. Inst. Steklov. (POMI) 410 (2013), 25-35. MR3048260

[5] A. A. Kiselev and O. A. Ladyzhenskaya, On the existence and uniqueness of the solution of the nonstationary problem for a viscous, incompressible fluid, Izv. Akad. Nauk SSSR Ser. Mat. 21 (1957), no. 5, 655-680. (Russian) MR0100448 (20:6881)

[6] G. Koch, N. Nadirashvili, G. A. Seregin, and V. Sverák, Liouville theorems for the Navier-Stokes equations and applications, Acta Math. 203 (2009), no. 1, 83-105. MR2545826(2010i:35281)

[7] H. Koch and V. A. Solonnikov, $L_{p}$-estimates for a solution to the nonstationary Stokes equations. Function theory and phase transitions, Probl. Mat. Anal., No. 22, Nauch. Kniga, Novosibirsk, 2001, pp. 197-239; English transl., J. Math. Sci. (N. Y.) 106 (2001), no. 3, 3042-3072. MR.1906033 (2003c:35132)

[8] O. A. Ladyzhenskaya, Mathematical problems of the dynamics of viscous incompressible fluids, 2nd ed., Nauka, Moscow, 1970. (Russian)

[9] J. Leray, Sur le mouvement d'un liquide visqueux emplissant l'espace, Acta Math. 63 (1934), no. 1, 193-248. MR 1555394

[10] G. A. Seregin, Local regularity of suitable weak solutions to the Navier-Stokes equations near the boundary, J. Math. Fluid Mech. 4 (2002), no. 1, 1-29. MR1891072 (2003a:35152)

[11] _ A note of local boundary regularity for the Stokes system, Zap. Nauchn. Sem. S.-Peterburg. Otdel. Mat. Inst. Steklov. (POMI) 370 (2009), 151-159. English transl., J. Math. Sci. (N. Y.) 166 (2010), no. 1, 89-90. MR2749216 (2012a:35251) 
[12] G. A. Seregin and V. Šverák V., On type I singularities of the local axi-symmetric solutions of the Navier-Stokes equations, Comm. Partial Differential Equations 34 (2009), no. 1-3, 171-201. MR2512858(2010k:35356)

[13] V. A. Solonnikov, Estimates of the solutions of the non-stationary Navier-Stokes system, Zap. Nauchn. Sem. Leningrad. Otdel. Mat. Inst. Steklov. (LOMI) 38 (1973), 46-93. (Russian) MR0415097 (54:3188)

[14] V. A. Solonnikov, On nonstationary Stokes problem and Navier-Stokes problem in a half space with initial data nondecreasing at infinity, Probl. Mat. Anal., No. 25, Nauch. kniga, 2003, pp. 189-210; English transl., J. of Math. Sci. (N.Y.) 114 (2003), no. 5, 1726-1740. MR.1981302 (2004h:35183)

[15] _ Estimates for solutions of the nonstationary Stokes problem in anisotropic Sobolev spaces and estimates for the resolvent of the Stokes operator, Uspekhi Mat. Nauk 58 (2003), no. 2, 123-156; English transl., Russian Math. Surveys 58 (2003), no. 2, 331-365. MR1992567(2004h:35184)

[16] E. Stein, Harmonic analysis: real-variable methods, orthogonality and oscillatory integrals, Princeton Univ. Press, Princeton, NJ, 1993. MR1232192 (95c:42002)

\section{OXFord University, United Kingdom}

St. Petersburg Branch, Steklov Mathematical Institute, Russian Academy of Sciences, Fontanka 27, 191023 St. Petersburg, Russia

E-mail address: seregin@pdmi.ras.ru

UNIVERSITY OF MinNESOTA

E-mail address: sverak@math.umn.edu

Received 7/JAN/2013

Originally published in English 